\title{
Detection of Chikungunya Virus in Saliva and Urine Samples of Patients from Rio de Janeiro, Brazil. A Minimally Invasive Tool for Surveillance
}

\section{Tiago Souza Salles}

Universidade Federal do Rio de Janeiro Instituto de Quimica

Thayane Encarnação Sá-Guimarães

Universidade Federal do Rio de Janeiro Instituto de Quimica

Victor Guimarães-Ribeiro

Universidade Federal do Rio de Janeiro Instituto de Quimica

Ana Claudia Amaral Melo

Universidade Federal do Rio de Janeiro

Davis Fernandes Ferreira

Universidade Federal do Rio de Janeiro

Monica Ferreira Moreira ( $\triangle$ monica@iq.ufrj.br)

Universidade Federal do Rio de Janeiro Instituto de Quimica https://orcid.org/0000-0002-3720-415X

Short report

Keywords: Chikungunya virus, saliva and urine samples, epidemiological surveillance of arbovirus, SYBR Green assay

Posted Date: February 9th, 2021

DOI: https://doi.org/10.21203/rs.3.rs-178829/v1

License: (c) (1) This work is licensed under a Creative Commons Attribution 4.0 International License.

Read Full License 
1 Detection of Chikungunya virus in saliva and urine samples of patients

2 from Rio de Janeiro, Brazil. A minimally invasive tool for surveillance

3 Tiago S. Salles ${ }^{1,2}$, Thayane E. Sá-Guimarães ${ }^{1}$, Victor Guimarães-Ribeiro ${ }^{1}$, Ana Claudia A. Melo ${ }^{1,2}$, Davis F. Ferreira ${ }^{3,4}$ and *Monica F. Moreira ${ }^{1,2}$

7 1-Federal University of Rio de Janeiro, Laboratory of Biochemistry and

8 Molecular Biology of Vectors, Institute of Chemistry, Rio de Janeiro, RJ, Brazil.

9 2-National Institute of Science and Technology in Molecular Entomology, Brazil.

10 3- Federal University of Rio de Janeiro, Institute of Microbiology, Rio de Janeiro, 11 RJ, Brazil.

12 4- Department of Molecular and Structural Biochemistry, North Carolina State 13 University, Raleigh, NC, USA.

${ }^{*}$ Corresponding Author

16 Address: Laboratório de Bioquímica e Biologia Molecular de Vetores,

17 Departamento de Bioquímica, Instituto de Química, Caixa Postal 68563, 18 Universidade Federal do Rio de Janeiro, Rio de Janeiro, RJ, 21941-909, Brasil

19 Telephone Number: +55213938 7365

20 Fax Number: +55213938 7266

21 E-mail: monica@iq.ufri.br 


\section{Abstract}

26 In this study, we collected saliva and urine samples from individuals in the

27 metropolitan region of Rio de Janeiro, Brazil, during the years of 2017 through

282019 and we were able to detect the presence of Chikungunya virus genome in

29 these samples. Our findings reinforce the possibility to monitor Chikungunya virus

30 circulation by analyzing saliva and urine from individuals during inter-epidemic 31 periods.

Keywords: Chikungunya virus, saliva and urine samples, epidemiological surveillance of arbovirus, SYBR Green assay.

Chikungunya virus (CHIKV) is a mosquito-borne virus, transmitted in the urban area mainly by the Aedes aegypti and Aedes albopictus mosquitoes[1]. CHIKV belongs to family Togaviridae and genus Alphavirus and consists of a singlestranded RNA genome virus, about $70 \mathrm{~nm}$ diameter and a phospholipid envelope[2]. This virus was first described in Africa (Tanzania) in 1954 and later identified in Asia, and it was responsible for outbreaks in these two continents

42 from the 1960s to the 1980s [3]. Currently, CHIKV is considered a real threat to countries localized in temperate and tropical zones that are infested by Aedes spp, such as Europe and the Americas [3,4]. 
Human CHIKV infection results in a spectrum of manifestations and it begins with

a silent incubation period lasting 2-4 days on average (range 1-12 days) [5], which can evolve to either an asymptomatic and subclinical outcome or to clinical manifestations. Clinical onset is abrupt, with high fever, headache, back pain, myalgia, and arthralgia; the latter can be intense, affecting mainly the extremities (ankles, wrists, phalanges) but also large joints, referred to as the effect of the 52 incapacitating arthralgia [6].

Our group collected saliva and urine from volunteers presenting symptoms compatible with the disease, except for one healthy individual (male) from which saliva was collected. The individuals that had saliva collected were one male and 57 four females. The individuals that had urine collected were two males and four females. Three females donated both, saliva and urine. The samples were collected from individuals at the Federal University of Rio de Janeiro, using approved Protocol Ethics: 80709 HUCFF/FM/UFRJ from 2017 through 2019. Volunteers were students, student relatives and staff from the University Campus, and the ages were between 25 and 45 years old.

63

64 The saliva samples $(n=5)$ were obtained from five individuals with arbovirus-like symptoms and one asymptomatic individual. In the urine group ( $\mathrm{n}=6$ ) all 66 individuals were exhibiting arbovirus-like symptoms. The total number of 67 samples, therefore, was eleven $(n=11)$ and the total number of individuals was eight $(n=8)$. Individual samples were submitted to RNA extraction by the TRIzol 
TM method Reagent (Invitrogen, Carlsbad, California, USA), followed by a

70 Reverse Transcriptase (RT) assay by superscript IV (Invitrogen, Carlsbad,

71 California, USA), following the manufacturer recommendation. CHIKV RNA

72 detection was performed by using RT 2-step-qPCR and conventional PCR, using

73 the forward 5'-ctttggagccaacgctatcgett-3' (SGCK-F) and reverse 5'-

74 tttgtccttgcactctgctgta-3' (SGCK-R) primers in the standardization SYBR Green

75 qPCR assays. For the conventional PCR, Nested methodology was used in the

76 first reaction with the forward 5'-taccgtataagactctagtc-3' (Nestalpha-F) and reverse 5'-tgaatgtccccaaatcttccagg-3' (Nestalpha-R) primers, followed by the second reaction with primers (SGCK-F/SGCK-R). The amplified viral material was visualized on a 1.5\% (v/v) agarose gel (Fig. 1D).

80

81 The standardization of the SYBR GREEN qPCR assay showed a efficiency of 93.1\% and specific for the detection of CHIKV genetic material as shown in (Fig. $1 \mathrm{~A}$ and $1 \mathrm{~B}$, Table $1 \mathrm{~A})$. The $\mathrm{qPCR}$ results showed that all urine samples were positive for CHIKV RNA, including the sample from the asymptomatic individual.

85 Four saliva samples were positive, and one was negative. The negative saliva sample was from the donor that had arbovirus-like symptoms but donated saliva only. All two individuals that had symptoms and donated both saliva and urine had both samples positive. Overall, we had ten positive samples for CHIKV and one negative (Fig. 1E and Table 1B). The generic RT-PCR for alphaviruses showed positive results, and direct sequencing of the viral amplicons showed a CHIKV-specific sequence. The result of the Sanger sequencing was used as 
92 query for BLAST searches of NCBI. In order to identify query sequences were used to search sequences on the CHIKV genome on the NCBI genome database, using BLASTn [7]. Sequences resulting from these searches, showing more than $90 \%$ identity were deemed as homologous. All the sequences from the homologous were then used as Datasets to the aligned using the MUSCLE software [8]and then pruned for removal of regions with a high frequency of indels using TrimAL using the "-gappyout" comman [9].

The maximum likelihood $(\mathrm{ML})$ tree topology was inferred with the lq-Tree 1.6 program [10]. Branch support was assessed by the ultrafast bootstrap implementation of IqTree using 1,000 replicates [11]. IqTree was executed via 102 the command "iqtree -s infile -bb 1000". Because no outgroup was included in our analysis, rooting of the genome CHIKV genealogy was performed using the minimal ancestor deviation method of Tria at al. [12]. Fig 2 shows the phylogenetic tree indicating in red the RIO_2019_CHIKV genome sequenced in the work. Our results are similar to those of Musso et. al. [13] where they reported

107 high number of CHIKV-positive samples during a period of high CHIKV 108 prevalence, agreeing with the epidemiological data of the metropolitan region of 109 Rio de Janeiro at the time of collection [4,14].

111 In the context of endemic co-circulation of other arbovirus diseases transmitted

112 by $A$ e. aegypti such as dengue and zika fevers [14,15], a fast, reliable and 113 noninvasive diagnostic method is important for surveillance and early detection 114 of an increase in the number of infections. In this study, we show that it is possible 
115 to detect CHIKV in saliva and urine samples of symptomatic and asymptomatic

116 individuals. The time of sample collection in this study correlated to an outbreak

117 in Rio de Janeiro city. Considering the endemicity of CHIKV in the state, our

118 results strengthen the use of saliva (preferable) and urine as important tools for

119 detecting asymptomatic individuals during inter-epidemic and pre-epidemic 120 periods.

121

122 Ethical approval

123 The Ethical Committee of RJ/BR Rio de Janeiro (80709 HUCFF/FM/UFRJ) has

124 approved this study.

125

126 Declaration of interest

127 Declarations of interest: none

128

129 Author contributions

130 All authors collaborated in the study design; TSS, TESG, VGR, participated in

131 diagnosing and managing these eight patients; TSS, ACAM, and MFM

132 participated in epidemiological investigation; TSS, DFF, and MFM extracted and

133 analyzed the clinical data; TSS and VGR prepared the first manuscript draft;

134 DFF, ACAM and MFM modified the manuscript subsequently; all authors have

135 reviewed and approved the final manuscript.

136

137 Acknowledgments 
138 The authors are grateful to the Program for Technological Development in Tools 139 for Health-RPT-FIOCRUZ for use of its facilities.

\section{Formatting of funding sources}

142 This work was supported by the Fundação Carlos Chagas Filho de Amparo

143 à Pesquisa do Estado do Rio de Janeiro (FAPERJ-E-26/201.331/2016-REDE

144 ZIKA), Conselho Nacional de Desenvolvimento Científico e Tecnológico

145 (CNPq), Instituto Nacional de Ciência e Tecnologia em Entomologia Molecular

146 (INCT-EM/CNPq), and Coordenação de Aperfeiçoamento de Pessoal de Nível

147 Superior (CAPES).

149 Reference

150 1. Sergon K, Njuguna C, Kalani R, Ofula V, Onyango C, Konongoi LS, et al.

151 Seroprevalence of Chikungunya virus (CHIKV) infection on Lamu Island, Kenya, October 152 2004. American Journal of Tropical Medicine and Hygiene. 2008;78:333-7.

153 2. Strauss JH, Strauss EG. The alphaviruses: gene expression, replication, and evolution.

154 Microbiological Reviews [Internet]. 1994;58:491 LP - 562. Available from:

155 http://mmbr.asm.org/content/58/3/491.abstract

156 3. Simon F, Savini H, Parola P. Chikungunya: A Paradigm of Emergence and

157 Globalization of Vector-Borne Diseases. Medical Clinics of North America. 2008. p.

158 1323-43.

159 4. Maljkovic Berry I, Rutvisuttinunt W, Sippy R, Beltran-Ayala E, Figueroa K, Ryan S, et

160 al. The origins of dengue and chikungunya viruses in Ecuador following increased

161 migration from Venezuela and Colombia. BMC Evolutionary Biology [Internet].

162 2020;20:31. Available from: https://doi.org/10.1186/s12862-020-1596-8

163 5. Pialoux G, Gaüzère BA, Jauréguiberry S, Strobel M. Chikungunya, an epidemic

164 arbovirosis. Lancet Infectious Diseases. 2007;7:319-27.

165 6. Enserink M. Massive Outbreak Draws Fresh Attention to Little-Known Virus. Science 166 [Internet]. 2006;311:1085 LP - 1085. Available from:

167 http://science.sciencemag.org/content/311/5764/1085.1.abstract

168 7. Morgulis A, Coulouris G, Raytselis Y, Madden TL, Agarwala R, Schäffer AA. Database 169 indexing for production MegaBLAST searches. Bioinformatics (Oxford, England).

170 2008;24:1757-64. 
8. Edgar RC. MUSCLE: multiple sequence alignment with high accuracy and high throughput. Nucleic acids research. 2004;32:1792-7. 9. Capella-Gutiérrez S, Silla-Martínez JM, Gabaldón T. trimAl: a tool for automated alignment trimming in large-scale phylogenetic analyses. Bioinformatics (Oxford, England). 2009;25:1972-3. 10. Trifinopoulos J, Nguyen L-T, von Haeseler A, Minh BQ. W-IQ-TREE: a fast online phylogenetic tool for maximum likelihood analysis. Nucleic Acids Research [Internet]. 2016;44:W232-5. Available from: https://doi.org/10.1093/nar/gkw256 11. Hoang DT, Chernomor O, von Haeseler A, Minh BQ, Vinh LS. UFBoot2: Improving the Ultrafast Bootstrap Approximation. Molecular biology and evolution. 2018;35:51822. 12. Tria FDK, Landan G, Dagan T. Phylogenetic rooting using minimal ancestor deviation. Nature ecology \& evolution. England; 2017;1:193. 13. Musso D, Teissier A, Rouault E, Teururai S, de Pina JJ, Nhan TX. Detection of chikungunya virus in saliva and urine. Virology Journal [Internet]. Virology Journal; 2016;13:1-4. Available from: http://dx.doi.org/10.1186/s12985-016-0556-9 14. Ministério da Saúde. Monitoramento dos casos de Arboviroses urbanas transmitidas pelo Aedes (dengue, chikungunya e Zika). Boletim Epidemiológico Arboviroses [Internet]. 2019;51:1-13. Available from: https://portalarquivos2.saude.gov.br/images/pdf/2020/janeiro/20/Boletimepidemiologico-SVS-02-1-.pdf 15. Salles TS, da Encarnação Sá-Guimarães T, de Alvarenga ESL, Guimarães-Ribeiro V, de Meneses MDF, de Castro-Salles PF, et al. History, epidemiology and diagnostics of dengue in the American and Brazilian contexts: a review. Parasites \& Vectors [Internet]. 2018;11:264. Available from: https://doi.org/10.1186/s13071-018-2830-8

Table 1 - standardization and amplification of samples by qPCR SYBR GREEN assay

\begin{tabular}{|c|c|}
\hline \multicolumn{2}{|c|}{$\begin{array}{l}\text { A) performance and Standardization for the SYBR GREEN assay } \\
\text { using Chikungunya virus with specific primers (SGCK-F / SGCK- } \\
\text { R) }\end{array}$} \\
\hline Virus dilution \# & Average $C T$ in triplicate \\
\hline
\end{tabular}




\begin{tabular}{ll}
\hline $1: 10$ & 15,54 \\
$1: 100$ & 17,48 \\
$1: 1000$ & 20,85 \\
$1: 10000$ & 24,47 \\
$1: 100000$ & 28,19 \\
$1: 1000000$ & 31,92 \\
$1: 10000000$ & 36,79 \\
\hline $\mathrm{H}_{2} \mathrm{O}$ & N/A \\
\hline
\end{tabular}

B) amplification for the SYBR GREEN assay using Chikungunya virus, saliva and urine samples with the specific primers (SGCKF/SGCK-R)

\begin{tabular}{|c|c|c|}
\hline Sample code & $\begin{array}{c}\text { Sample } \\
\text { types }\end{array}$ & Average $C T$ in triplicate \\
\hline 16 & saliva & $\mathrm{N} / \mathrm{A}$ \\
\hline 44 & saliva & 31,74 \\
\hline 45 & urine & 33,41 \\
\hline 46 & saliva & 33,11 \\
\hline 47 & urine & 36,69 \\
\hline 48 & urine & 35,88 \\
\hline 49 & saliva & 36,02 \\
\hline 50 & urine & 35,61 \\
\hline 51 & saliva & 34,65 \\
\hline 52 & urine & 35,84 \\
\hline 53 & urine & 35,85 \\
\hline Positive control \# & $1: 10$ & 14,97 \\
\hline Positive control \# & $1: 1000$ & 22,71 \\
\hline
\end{tabular}




\begin{tabular}{|c|c|c|}
\hline Negative control \# & - & N/A \\
\hline $\mathrm{H}_{2} \mathrm{O}$ & - & N/A \\
\hline \multicolumn{3}{|c|}{${ }^{*}$ cDNA from the Vero cell supernatant } \\
\hline \multicolumn{3}{|c|}{ * cDNA from the CHIKV suspension } \\
\hline
\end{tabular}

\section{Legend}

Fig.1:(A) Amplification curve for the qPCR standardization and sensitivity determination and (B) Melting pattern of the serial dilution curve for the CHIKV cDNA for the standardization with the SGCK-F / SGCK-R primers for qPCR with SYBR Green. (C) Amplification curve for the urine and saliva samples and (D)

Melting pattern of the serial dilution curve for the CHIKV cDNA (positive control), saliva and urine cDNA samples for quantification with the SGCK-F / SGCK-R primers for qPCR. (E) Linear regression for the serial dilution curve with the CHIKV cDNA for standardization with the SGCK-F / SGCK-R primers in qPCR with SYBR Green, which made it possible to determine the efficiency of the reaction in the value of $93,1 \%$. (F) qPCR gel for the cDNAs standard CHIKV cDNA with the SGCK-F / SGCKR primers; MM: molecular mass; B: blank; CHIKV, Chikungunya virus; MAYV, Mayaro virus; SINDV, Sindbis virus; FLAV,

214 pool of flavivirus (DENV1-4, YFV, ZIKV). (G) qPCR gel for the CHIKV cDNAs

215 (saliva and urine) and standard CHIKV cDNA with the SGCK-F / SGCKR primers; MM: molecular mass; B: blank; red: saliva; blue: urine samples 
218 Fig 2. Phylogenetic tree using the sequences searched in BLASTn as dataset to 219 alignment in MUSCLE software and using IQTREE to the construction of the 220 phylogenetic tree, highlight in red the RIO2019CHIKV genome sequenced this 221 work. 

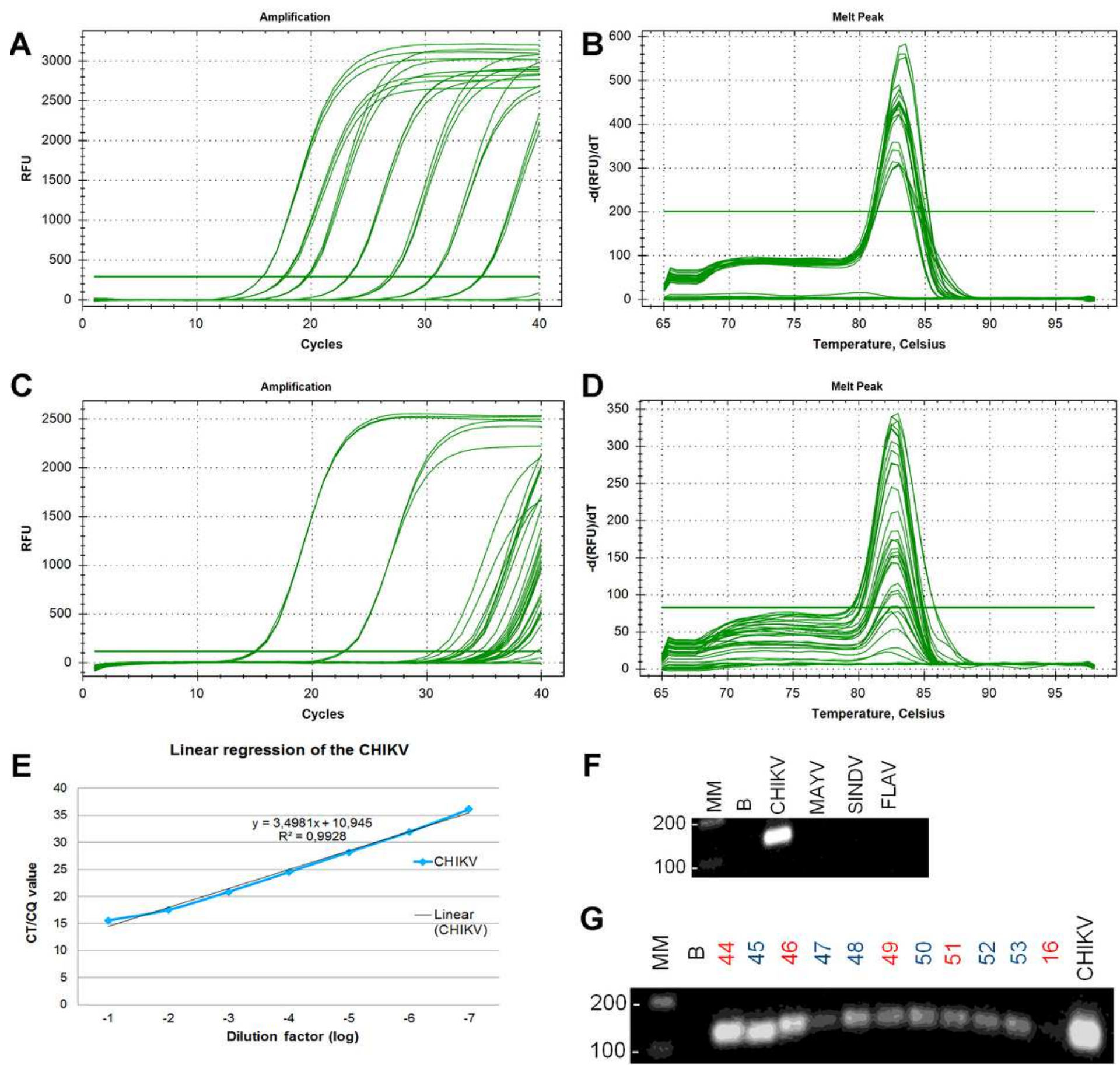

\section{Figure 1}

(A) Amplification curve for the qPCR standardization and sensitivity determination and (B) Melting pattern of the serial dilution curve for the CHIKV cDNA for the standardization with the SGCK-F / SGCK-R primers for qPCR with SYBR Green. (C) Amplification curve for the urine and saliva samples and (D) Melting pattern of the serial dilution curve for the CHIKV cDNA (positive control), saliva and urine CDNA samples for quantification with the SGCK-F / SGCK-R primers for qPCR. (E) Linear regression for the 
serial dilution curve with the CHIKV cDNA for standardization with the SGCK-F / SGCK-R primers in qPCR with SYBR Green, which made it possible to determine the efficiency of the reaction in the value of $93,1 \%$. (F) qPCR gel for the cDNAs standard CHIKV cDNA with the SGCK-F / SGCKR primers; MM: molecular mass; B: blank; CHIKV, Chikungunya virus; MAYV, Mayaro virus; SINDV, Sindbis virus; FLAV, pool of flavivirus (DENV1-4, YFV, ZIKV). (G) qPCR gel for the CHIKV cDNAs (saliva and urine) and standard CHIKV cDNA with the SGCK-F / SGCKR primers; MM: molecular mass; B: blank; red: saliva; blue: urine samples

Tree scale:

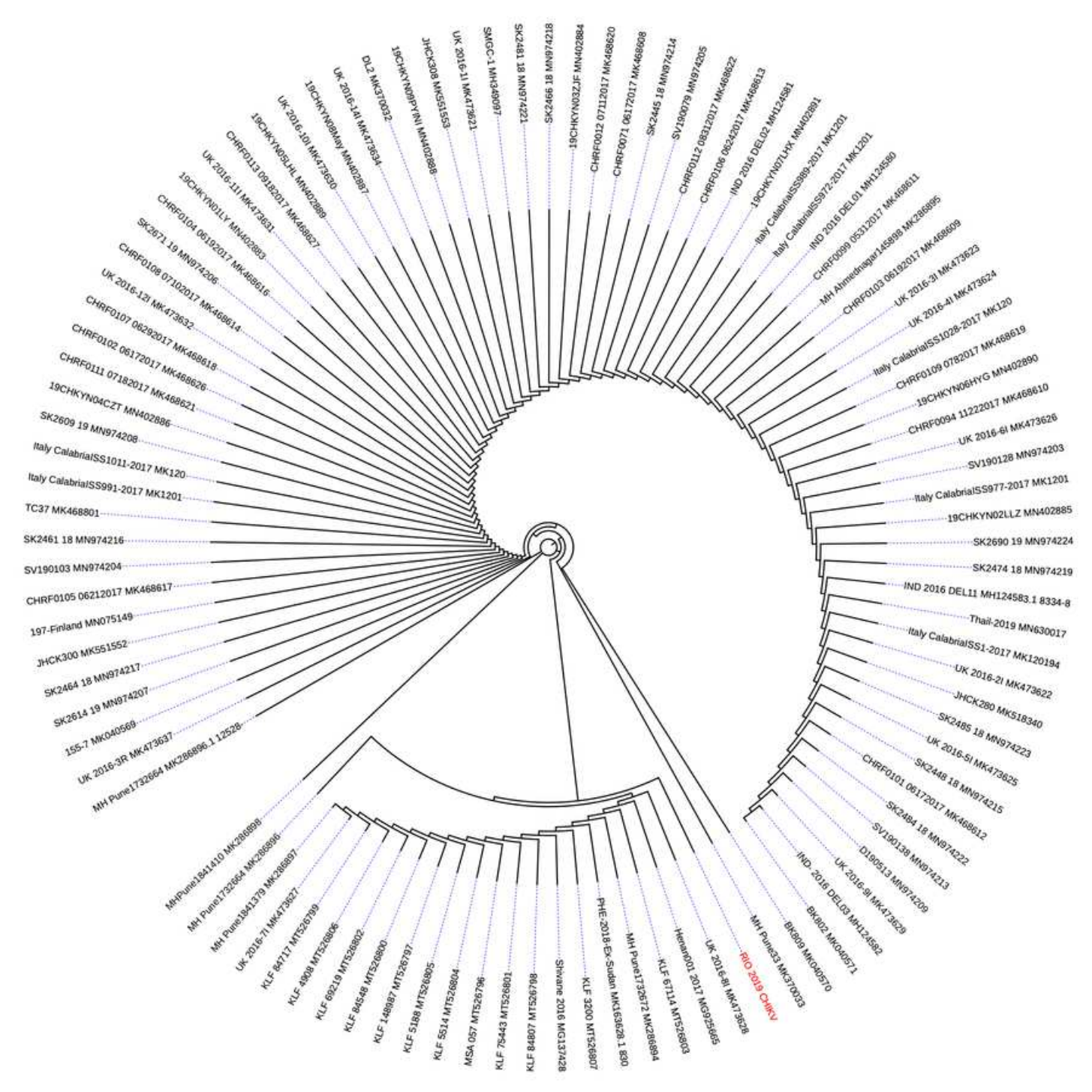

Figure 2

Phylogenetic tree using the sequences searched in BLASTn as dataset to alignment in MUSCLE software and using IQTREE to the construction of the phylogenetic tree, highlight in red the RIO2019CHIKV genome sequenced this work. 